\title{
Less is More: Analysing Communication in Teams of Strangers
}

\author{
Evelyn T S Tan \\ University of York \\ ett506@york.ac.uk \\ Georgia Heyes \\ University of York \\ gh882@york.ac.uk
}

\author{
Alex Wade \\ University of York \\ alex.wade@york.ac.uk \\ Simon Demediuk \\ University of York \\ simon.demediuk@york.ac.uk
}

\author{
Athanasios Kokkinakis \\ University of York \\ athanasios.kokkinakis@york.ac.uk \\ Anders Drachen \\ University of York \\ anders.drachen@york.ac.uk
}

\begin{abstract}
Teamwork is challenging in cooperative digital games, especially between strangers. In many online cooperative games, teams have a short-lived existence and ever-changing membership. Our study explores how short-lived, ad hoc teams of strangers communicate and investigate its effect on team performance. We use the commercial cooperative digital game, Portal 2 and analyse 2256 text message instances produced by teams during a 45-minute interaction. Our findings show that team communication is negatively related to performance, and affects performance over and beyond prior experience. A content analysis shows that teams generally have higher task-related communication than socio-emotional communication. This pattern is consistent throughout the duration of the interaction period. The results are discussed in the context of previous research on team communication and performance, and we draw parallels with communication patterns in real-world groups such as aviation crews.
\end{abstract}

\section{Introduction}

Digital games have evolved in waves over the past forty years. From the first, simple, text-based adventures, contemporary games have transformed into a plethora of types and genres to accommodate a vast global and inter-generational audience [1]. Today, games span the range from the original text-based format to three-dimensional worlds covering thousands of square kilometres of virtual real estate with millions of people interacting online.

Besides their entertainment purpose, digital games have become an avenue for people to develop social connections and make new ones [2, 3, 4]. For example, after lockdown measures were introduced globally during the start of the 2020 coronavirus pandemic,
Microsoft reported a $130 \%$ increase in multiplayer gaming and 23 million new friendship connections made over their Xbox Live service from March to April 2020 [5]. Additionally, a recent report shows that multiplayer titles, especially those with cooperative elements, are well-represented in the 20 most popular PC games [6]. This shows how important the social aspects of digital games, e.g. interacting with others in some way, are to game success.

Our study focuses on cooperative games. Cooperative games range from simple two-player, fully cooperative environments like Portal 2 and $I b b \&$ $O b b$, to more complex games with teams of five or six players in mixed cooperative-competitive environments like League of Legends and Counter Strike. Regardless of the complexity, or presence of competitive elements, one of the core challenges presented by cooperative games is to effectively work together.

Within cooperative games, our study specifically investigates online teams of strangers. With over 2.5 billion players worldwide [7], playing with strangers is common in online multiplayer games. In cooperative games, working effectively as a team of strangers is challenging. The familiarity that comes with preexisting relationships makes it easier to predict the actions, behaviours and mental models that a teammate might have. However, when there is no familiarity, teams of strangers can easily run into problems of coordination and communication $[8,9]$.

Due to the large player base, many online cooperative games employ some form of automatic matchmaking to help players find teams and join games quickly [10]. As a result, a team's existence is usually constrained by the duration of the game. This short-lived existence (e.g. 15-45 minutes) further challenges teams' ability to develop processes that facilitate effective teamwork. This unique context invites questions about the underlying mechanisms supporting effective teamwork in ad hoc teams of strangers.

To this end, we investigate team communication 
between strangers, specifically text chat, using the commercial puzzle-solving game Portal 2 [11]. We focus on team communication because it is necessary for teamwork - it is the means by which team members combine their knowledge, coordinate actions, and develop affective relations [12, 13]. As such, it provides an index of the emerging dynamics in the team and its subsequent impact on performance and personal relations. We selected Portal 2 (description in Section 3) because it allows us to focus on the communication between two strangers in a controlled manner. Since Portal 2 focuses solely on going from point A to point B without distractions, any observed communication effects can be more clearly attributed to player behaviour. By investigating team communication in this context, we hope to advance our understanding of what makes effective ad hoc teams of strangers.

\section{Related Work}

Communication and performance: Team communication as a predictor of performance has been studied widely outside of digital games. Theoretically, communication enables team members to send and receive information regarding the environment, to formulate strategies, make decisions and coordinate actions [14]. Its role in enabling team processes makes team communication a key determinant of performance. This has been confirmed in a recent meta-analysis on 150 studies containing over 9000 teams, showing that communication is significantly and positively $(\rho=0.31$, $95 \%$ CI $[0.23,0.30])$ related to team performance [15]. Although the meta-analysis did not include teams in digital games, we expect to find a similar relationship since a variety of team types were included in the analysis.

\section{H1: Team communication will be positively related to performance}

Communication content: The relationship between communication and performance might be moderated by the communication content. While there are nuances, communication in groups can generally be categorised as task-oriented or socio-emotional (or relational) [16]. Socio-emotional content develops the climate or atmosphere within a team, which can have an influence on teams' progress on a task, but task-oriented content is likely to have a direct link as it pertains to 'getting the job done' [14]. Thus, we hypothesise:

H2: Task-oriented communication will be more strongly related to performance compared to socio-emotional communication.
In teams of strangers with short-lived interactions, we also expect communication to be mostly task-oriented. Previous research investigating the communication content in digital game teams has found higher socio-emotional compared to task-oriented communication $[17,18,19]$, owing to the recreational nature of games. Socio-emotional content made up $50 \%$ of communication in a study of Counter Strike teams, with $25 \%$ of content relating to game strategy [17]. Similar findings were observed in a study of Jedi Knight II players [18]. However, we expect the opposite pattern because our study focuses on teams of strangers, whereas these studies have investigated long-term groups and teams. When future interaction is expected, individuals tend to engage in more positive, and relational forms of communication [20].

Although digital games are inherently recreational and playful environments, the formation and life span of the team will influence social interactions. Research on zero-history computer-mediated work groups have generally observed higher task-oriented communication if the team has been formed for a short-term, time-sensitive purpose [20]. Parallels can also be drawn with real-world teams with similar structure (i.e. zero-history, short-lived, time sensitive), such as crisis teams and airline crews.

The higher levels of task-oriented communication observed in these 'swift starting action teams' is attributed to the task- and performance-driven environment that the teams operate in [21, 22]. These teams work under evident time pressure and have to perform almost immediately upon formation. While the playful nature of games suggests that higher levels of socio-emotional content would be observed in teams of strangers in digital games, we expect that the short-lived nature of the team functioning in a problem solving environment (Portal 2) will engender higher task-oriented communication.

H3: Team communication will be more task-oriented than socio-emotional

\section{Method}

Participants: 66 participants, comprising university students and staff members, were recruited via advertisements and word of mouth. The call-for-participants advertisement noted that the study was open to people with any experience level of digital games and was not exclusively focused on 'gamers'.

23 participants identified as female, 1 identified as non-binary, and 42 identified as male $(M=21.9$ years old, $S D=6.51$, range $=18-64$ years old). Participants 
were placed in 33 two-person teams, where they were matched based on availability, and where possible, their level of experience with the game.

Level of experience was based on the number of hours participants reported previously having played either Portal 1 or Portal 2. In almost half the sample $(42 \%)$, the more experienced participant in the team had spent at least $50 \%$ more time on the game than the less experienced participant.

Portal 2 Cooperative Mode: Portal 2 is a three-dimensional, 'first person perspective' puzzle-solving digital game. It has a single player mode and a two-player, cooperative mode. This study refers to the cooperative mode when using the term Portal 2.

Portal 2 is easy to learn. As such, it is suitable for people with varying levels of experience with digital games. In Portal 2, each level is a puzzle. Successfully solving each puzzle means that the team is able to get from the start to the end of the level. Throughout the levels, players need to move items to specific positions, time their actions and move in turns. Players use 'portals' to carry out these actions (Figure 1). Hence, advancing through the levels require cooperation, communication and coordination. This allows us to investigate dyadic social interactions in a relatively naturalistic manner.

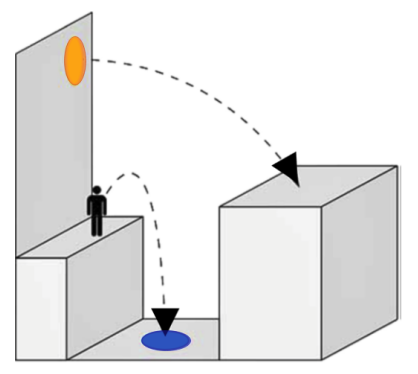

Figure 1. Adapted from Schute, Ventura and Ke [23]. Illustration of the core mechanic in Portal 2 creating portals. Players create two separate portals that allow them to move between surfaces: a blue portal to enter and a yellow portal to exit.

There are three ways to communicate in Portal 2 using text chat, voice chat and in-game communication mechanics like'pings', and 'gestures'. 'Pings' are semantically imbued, task-oriented, attention-focusing tools while 'gestures' are socio-emotional animations [24]. For this study, participants were only allowed to communicate using text chat and in-game communication mechanics. This was to ensure full anonymity between participants. This paper focuses on text chat analysis.
Procedure: This study was approved by the Ethics Committee of the Department of Psychology. The goal of the experimental setup was to maintain full anonymity between players, similar to the situation in online digital games. To achieve this, team members were emailed separately and were told different arrival times. This was to minimise the chance of players encountering each other in-person. Upon arrival, participants were sat in separate rooms with all windows covered.

Participants were given an information sheet detailing the aims of the study and provided informed consent. Participants were told that voice communication was disabled but that they could communicate with their teammate via text chat. Sticky notes containing information about controls such as moving, jumping, chatting and interacting with items in the game were stuck around the computer. This was to aid inexperienced participants.

All teams started from the Portal 2 tutorial level and were given 45 minutes to play through the game. Upon completion, participants were thanked and were shown out of the lab separately.

Bales' Interaction Process Analysis (IPA): Text chat instances were coded using Bales IPA [16]. An 'instance' represents separate lines of text chat sent by a player (see Table 1).

Table 1. Sample of coded text chat instances. Each row is an instance and each instance is assigned a code that corresponds to the coding scheme.

\begin{tabular}{l|l|l}
\hline player & Sentence & Code \\
\hline 1 & go ahead & 4 \\
\hline 2 & k & 3 \\
\hline 1 & woo teamwork & 1 \\
\hline 2 & what are we doing? & 9 \\
\hline 1 & it only opens briefly & 6 \\
\hline
\end{tabular}

Bales IPA was developed to understand the underlying processes that drive group interaction [16]. It consists of 12 categories that make up 4 higher-order categories: (1) Socio-emotional: Positive Reactions, (2) Task area: Attempted Answers, (3) Task area: Attempted Questions, (4) Socio-emotional: Negative Reactions. During the years, Bales IPA has faced some criticism for not accounting for communication content that contains multiple processes [25]. We acknowledge this criticism; we selected it because its higher-order categories provide a succinct summary of the verbal communication processes observed during the interaction between strangers in a cooperative task. This enables us to understand how communication processes might affect team performance and the overall dynamics 
in the team. To mitigate the limitations of Bales IPA, communication instances were coded based on their primary purpose.

Bales IPA has also been found to be suitable for the digital game context, although it is missing some categories that are typically observed in computer-mediated interactions. For example, communication content that is intended to correct for message errors (e.g. typos) and communication content that is not immediately related to the task. Nonetheless, it has been successfully used to investigate the presence of socio-emotional and task-oriented communication produced by players in a multiplayer game [18]. 14 categories in total were used to code the text communication between strangers in Portal 2 (Figure 2).

\section{Results}

\subsection{Communication and Performance}

We first analysed the relationship between team communication and performance. Team communication was operationalised as communication frequency. Performance was operationalised as the number of levels completed by the team. The tutorial level was not included in this analysis because the level was meant to teach participants how to interact with the game environment. Levels that were partially completed were also not included. The mean levels completed was $\mathrm{M}=$ $6.15, \mathrm{SD}=4.26$.

A negative correlation between team communication and performance was observed, $\rho=-0.36, p<.038$ (Figure 4). While our findings provide support for the link between team communication and performance, the observed negative effect is opposite to the direction found in Marlow and colleagues' [15] meta-analysis. Hypothesis 1 (Team communication will be positively related to performance) is not supported. Note that a non-parametric correlation was used due to the differential distance between game levels in terms of difficulty (e.g. getting from level 10 to 11 is not the same as getting from level 1 to level 2).

\subsection{Communication Content}

To analyse the communication content, three coders independently coded all the data. One team was removed from the analysis because they did not produce any textual communication $(n=32)$. Fleiss' kappa showed that there was good agreement between the raters $\kappa=0.69$ (95\% CI, 0.68 to 0.70$), p<.005[26,27]$.

We ran a correlation to test Hypothesis 2 (Task-oriented communication will be more strongly
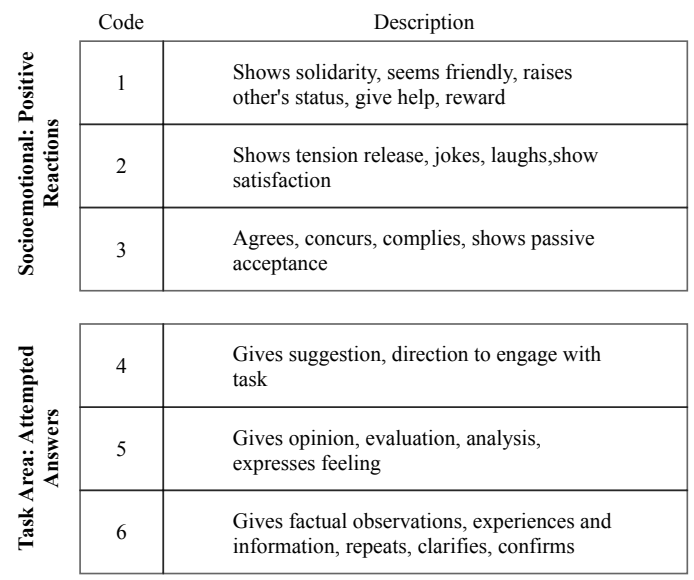

\begin{tabular}{|c|c|}
\hline 7 & $\begin{array}{l}\text { Asks for factual observations, experiences } \\
\text { and information, repeats, clarifies, confirms }\end{array}$ \\
\hline 8 & $\begin{array}{l}\text { Asks for opinion, evaluation, analysis, } \\
\text { expresses feeling }\end{array}$ \\
\hline 9 & $\begin{array}{l}\text { Asks for suggestion, direction to engage } \\
\text { with task }\end{array}$ \\
\hline
\end{tabular}

\begin{tabular}{|l|l|l|}
\cline { 2 - 3 } & 10 & $\begin{array}{l}\text { Disagrees, shows passive rejection, witholds } \\
\text { help }\end{array}$ \\
\cline { 2 - 3 } & & $\begin{array}{l}\text { Shows tension, confusion, frustration, asks } \\
\text { for help }\end{array}$ \\
\hline
\end{tabular}

\begin{tabular}{|c|l|}
\hline 13 & $\begin{array}{l}\text { communication outside of the immediate } \\
\text { task (e.g. have you had lunch?) }\end{array}$ \\
\hline
\end{tabular}

Figure 2. Description of codes used and the categories they represent. Code $1-12$ is based on Bales IPA, code 13-14 are additional categories for error corrections and non-task related conversation.

related to performance compared to socio-emotional communication). Task-oriented communication, $\rho$ $=-0.54, p<.001$ had a stronger correlation with performance than socio-emotional communication, $\rho$ $=-0.14, p<.446$, although the relationship between socio-emotional communication and performance is not significant. Hence, Hypothesis 2 is partially supported.

We then analysed the differences in communication content frequencies. Of the 2256 communication instances, the most frequent categories were giving suggestions/directions $(25 \%$, code 4), showing friendliness $(16 \%$, code 1$)$, sharing observations and experiences $(12 \%$, code 6$)$, and giving opinions $(11 \%$, code 5$)$. There was no negative, unfriendly or hostile communication (code 12) (see Figure 3). 


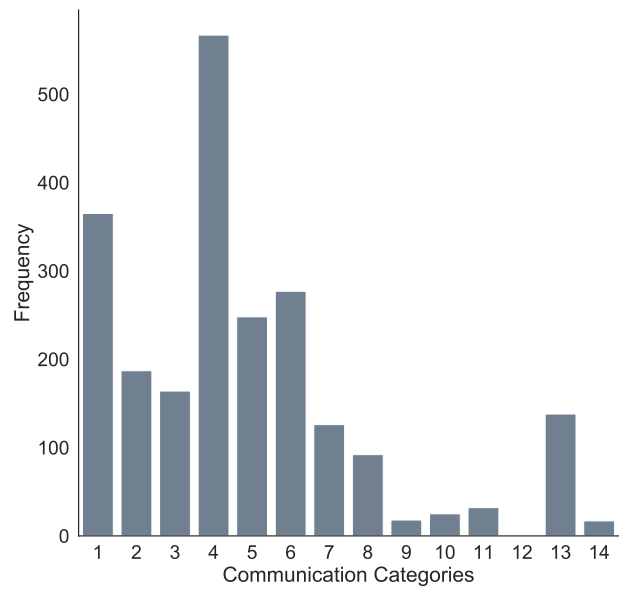

Figure 3. Frequency of communication categories.

When the codes are grouped into their respective higher-order categories (see Figure 2) we find that communication is most frequently used for task-related purposes (Table 2). The frequency of task-related communication (asking questions and giving answers) was almost double of socio-emotional communication (59\% vs 34\%), Hypothesis 3 (Team communication will be more task-oriented than socio-emotional) is supported.

Table 2. Distribution of Higher-Order Categories in Team Communication

\begin{tabular}{l|l}
\hline Category & \% total comms. \\
\hline Socio-emotional: Positive & $31 \%$ \\
\hline Task: Answers & $48 \%$ \\
\hline Task: Questions & $11 \%$ \\
\hline Socio-emotional: Negative & $3 \%$ \\
\hline Additional & $7 \%$ \\
\hline
\end{tabular}

\subsection{Additional Analyses}

Level of Experience, Performance and Communication: In addition to the hypothesis testing above, we explored the effect of experience on team performance. All 33 teams were included in this part of the analysis. Since we did not create novel Portal 2 chambers, participants with previous experience with the game will not only be more familiar with the mechanics, but will likely remember the solutions to the puzzles. This may influence team performance, communication and the relationship between communication and performance.

The level of experience in a team was operationalised as the sum of number of hours that each participant had previously spent on Portal (1
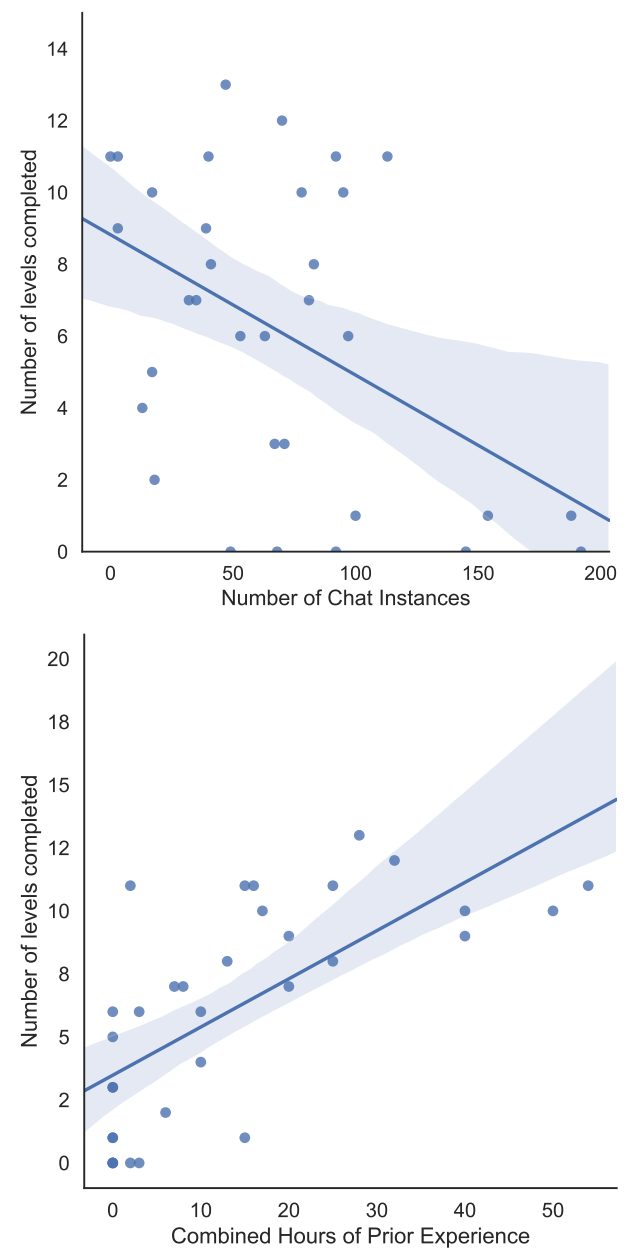

Figure 4. Top: A significant negative correlation between team communication and performance, $\rho=$ $-0.36, p<.038$. Bottom: A significant positive correlation between level of experience and performance, $\rho=0.76, p<.001$. 95\% confidence interval is indicated by shading around the line.

or 2). The mean level of experience (hours) in a team was $\mathrm{M}=13.97, \mathrm{SD}=15.38$. A strong and significant relationship between the level of experience in a team and performance was found $\rho=0.76, p<.001$ (Figure 4). The strong correlation suggests that the level of experience within a team might affect communication. However, no significant correlation between the level of experience within a team and volume of team communication was found.

Given the strong correlation between experience and performance, we investigated the effect of communication, controlling for level of experience. Since task-oriented communication had a stronger correlation to performance than total communication, we used only task-oriented communication for this 
analysis.

Before running the analysis, the independent variables were examined for collinearity. Results of the variance inflation factor (all less than 2.0), and collinearity tolerance (all above 0.8 ) suggest that multicollinearity was not a concern. An inspection of the P-P plot and the scatter plot of residuals indicate that the data also met the assumptions of normality and homoscedasticity. A hierarchical linear regression was run.

In the first step of the regression, only level of experience within a team was included as a predictor of performance. This model was statistically significant, $\mathrm{F}(1,31)=28.30, p<.001$, and explained $48 \%$ of the variance in performance. In the second step of the regression, level of experience within a team and task-oriented communication were included as predictors of performance. The second model was also statistically significant, $F(2,30)=22.85$, $p<.001$, and explained $60 \%$ of variance in performance. Task-oriented communication explained an additional $13 \%$ of variance in performance, after controlling for experience $\left(\mathrm{R}^{2}\right.$ Change $=0.126, \mathrm{~F}(1,30)=9.57, p$ $<.005)$. These findings indicate that communication contributes to team performance, over and above prior experience.
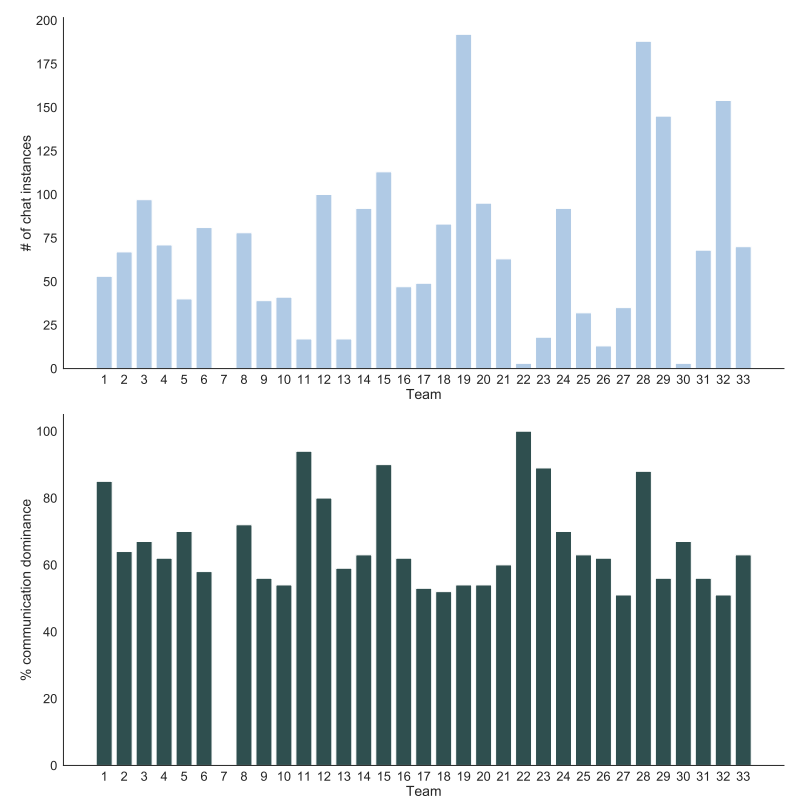

Figure 5. Comparison of total team communication and the percentage of communication attributed to the more talkative team member. Team 7 did not produce text chat.

\subsection{Communication Dynamics}

We then explored the communication dynamics. Teams differed in their volume of communication and levels of communication dominance. 20 teams had above $60 \%$ communication dominance by the more talkative player (see Figure 5), suggesting that, in general, one team member was producing more communicative instances than the other.

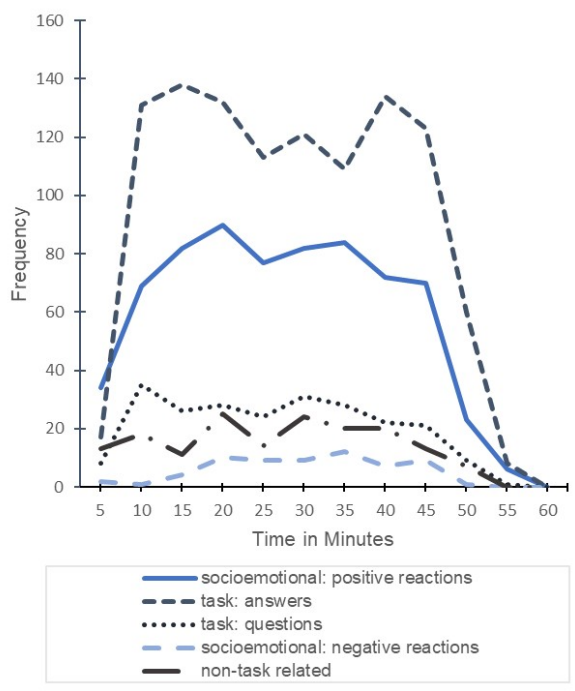

Figure 6. Frequency of higher-order communication categories over time. Communication related to giving instruction was the most frequent throughout the interaction, but high levels of positive reactions indicate a positive, cooperative team climate.

We also investigated whether the frequency of communication categories changed over time. One might expect task-oriented communication to decrease as teams become more familiar with the game mechanics. Similar to Section 4.2 Communication Content, only teams with text communication ( $n=$ 32) was used for this analysis. Figure 6 shows that the distribution of the frequency of categories remains relatively stable throughout the interaction. There is much more communication related to providing information compared to asking for information, and the valence of communication is highly positive. Together, these communication patterns indicate that team members are constantly giving unsolicited suggestions/information. The high level of friendliness and absence of negative communication indicates a positive and cooperative climate. 


\subsection{Summary}

In ad hoc teams of strangers, (text) communication and performance seem to be negatively linked, i.e. teams that communicate less perform better. Although the direction of effect is opposite to previous research [15], it supports the communication-performance relationship. Additional analysis showed that communication explains $13 \%$ of variance in performance in teams of strangers, above and beyond prior experience.

In line with observations of communication in real-world ad hoc teams, and zero-history computer-mediated work groups, we found higher levels of task-oriented communication compared to social communication. This is in contrast to previous research indicating that higher socio-emotional communication is expected due to the recreational, playful nature of digital games $[18,19]$.

Our exploratory analysis of communication dynamics indicated that team communication was dominated by one team member. Nonetheless, the high level of positive socio-emotional communication suggests that the climate in teams were generally positive and cooperative.

\section{Discussion}

By looking at team communication, our study takes a small step towards understanding the underlying mechanisms supporting effective teamwork in ad hoc teams of strangers in digital games. It also highlights the unique context of digital games - which has both recreational and performance-driven purposes - and whether theories of team effectiveness, which is largely based on work teams, applies.

Beyond digital games, our study highlights the value of off-the-shelf games as research tools for studying team dynamics. We show that by analysing communication, even during a short 45-minute interaction, rich and complex social interactions can be observed. These observations serve as objective indicators of the emerging dynamics in a team, and how it relates to various outcomes like performance. Using off-the-shelf games and conducting communication analysis are some of the recommendations that have been made to advance research on team process dynamics [28]. We discuss our findings in the next sections.

\subsection{Team Performance}

The strongest predictor of performance, accounting for $48 \%$ of variance, was the level of experience participants had with Portal 2. This is expected since we used default rather than custom levels. Participants with previous experience are familiar with the mechanics and may already know the solutions. This gives teams with more experienced players the upper hand.

However, team (task) communication explained an additional $13 \%$ of the variance. If this relationship is causal, it implies that improving task communication efficiency can positively impact performance. Indeed, this is the case for aviation crews where training resources have primarily been directed to improving team communication processes rather than improving individual expertise [21, 29]. This suggests that designing systems that help players communicate more efficiently may help players who are strangers work better together.

The direction of effect between communication and performance was surprising. A possible explanation for the negative relationship is the influence of the situation's temporal urgency, which was not included as a moderator in the communication-performance meta-analysis [15]. Since the experimental task was time sensitive, time spent deliberating reduced available time for executing actions.

Digital games also provide quick feedback loops on actions. Thus, teams with less communication might have higher performance because they spend more time trying possible solutions rather than discussing. Discussion may only be required when teams encounter difficulty and difficult levels impair performance. Toups and colleagues [30] made a similar observation in communication improvements of firefighting teams. An improvement in team coordination and performance was related to a reduction in verbal communication. Since the combined level of experience is strongly correlated with performance, it also suggests that in more experienced teams, players tend to type less and act more.

Another possible explanation for the direction of the relationship is that our analysis did not include in-game communication mechanics. Digital games have various in-game communication mechanics that allow players to communicate information by directly using the game system [24]. In-game communication mechanics allow players to communicate more efficiently [24]. For example, non-linguistic 'pings' are used to direct attention to a certain location in the environment and may convey meaning and intention.

In a study comparing the effect of different communication mediums on performance in Portal 2 , participants reported how in-game communication mechanics helped them elaborate information quickly [31]. On the contrary, explicit communication like 
text chat has high communication overhead - 'the cost in terms of time, cognitive bandwidth, and technological bandwidth of sharing information with other team members.' [24]. Although useful for explanations,conveying information via text chat takes longer than using in-game communication mechanics like 'pings', where information can be communicated using a single mouse click.. This suggests that the direction of the communication-performance relationship may be positive if in-game communication mechanics were included in the analysis. However, it might be that communication has a curvilinear relationship with performance such that communication is beneficial until teams know how to implicitly coordinate. Some evidence may be drawn from the team that was removed from the communication analysis. Despite having no text chat, the team completed 11 levels (the highest number of levels completed is 13).

\subsection{Communication Content}

In contrast to previous research on communication content during cooperative play [17, 18, 19], we found a higher volume of task-oriented communication compared to socio-emotional communication. It is possible that Pena and Hancock [18], who also used Bales IPA, observed the opposite trend (77\% socio-emotional communication, $23 \%$ task communication) because their sample were members of the same 'clan'. Clan members have ongoing relationships, which would manifest in the way they communicate with each other - exhibiting greater positive communicative behaviour to maintain cohesion and satisfaction within the group [32]. Hence the difference between our findings may be attributed to the absence of prior relations and absence of anticipation of future relations. Similar patterns have been observed in 'pick-up groups' in World of Warcraft [32] and are alluded to in an interview study of interacting with strangers in League of Legends [33].

The communication patterns in our findings more closely reflect the dynamics in 'swift starting action teams' such as airline crews [22] and military teams [34]. Communication tends to be highly task-oriented because these teams do not assume preexisting socio-emotional ties [21, 22, 34]. However, we suspect that the communication dynamics would be different if teams of strangers were not in a time sensitive, performance-focused environment. Hence, it is possible that the interaction between team characteristics - a team comprised of strangers, and task characteristics highly interdependent and requiring immediate action, may be driving the observed dynamics. Nonetheless, in digital games where ad hoc teams of strangers had to perform immediately, communication is likely to be predominantly task-oriented until the team disbands.

On a separate note, since digital games support the development of new social connections [5] and cooperation has been found to facilitate friendship development [35], this could mean that the degree to which strangers effectively collaborate might forge bonds that turn into friendship. Therefore, if we design systems that facilitate more effective collaboration between strangers, it could not only benefit performance but influence the development of social ties between players who are strangers.

\subsection{Communication Dynamics}

The high volume of communication related to giving information relative to communication related to asking for information throughout the interaction suggests that players may be giving unsolicited information. While this can be perceived and received negatively, the high level of positive socio-emotional communication throughout the game, indicates a positive team climate. This may be attributed to the environment - teams operated in a context with no competitive element, where cooperation was obviously the only route to success. Hence it is in the team's interest to foster a cooperative environment, which manifests as high levels of positive socio-emotional communication.

Another possibility is that early interactions had set the tone for subsequent interactions. When teams have no prior history, early interactions set the expectation for future behaviour [36]. These interactions tend to persist and have pronounced effects of subsequent communication [37, 38]. Jung and colleagues [38], for example, predicted team performance up to 6 months in advance using just 15 minutes of data on interaction dynamics from 30 teams. Given that digital games offer immediate feedback on actions, it is possible that the early positive and cooperative interactions has set the expectation for subsequent interactions.

\subsection{Limitations and Future Work}

By investigating communication dynamics, the work presented here extends previous research on the social interactions between strangers in team-based digital games but carries some limitations.

Firstly, although our sample size is larger than some previous studies examining communication in Portal 2 (e.g. $n=20$; [31]), it is still relatively small. A power analysis using $G^{*}$ Power [39] shows that a sample of 33 two-person teams, and effect size of $|0.36|$ for the 
correlation between communication and performance provides 0.57 power. Our findings should therefore be interpreted with caution and a larger sample size would improve statistical significance.

Our findings present only a partial picture of team communication dynamics since in-game communication mechanics were not incorporated into our analysis. By including the different modalities of communication, for example, voice chat and pings, a more comprehensive understanding of the relationship between communication and performance can be gained. For example, we hypothesise a positive relationship between 'pings' and team performance given that it reduces communication overhead, and allows information to be conveyed quicker than typing. Nonetheless, investigating text chat has provided some insight into the potential issues that inexperienced players face when using communication systems. It also highlights the type of communication that players would find most useful when working with strangers.

Our study also had a wide range of expertise which was useful in that it provided a wide spread of performance. However, it may have swamped the potential effects of personality and individual differences in communication ability. Further studies may try to use players with a smaller range of expertise - perhaps only recruiting novices. Having similarly experienced team members would enable us to address questions on how the level of expertise in a team influences communication patterns, as there is some evidence that it does [30]. These limitations imply that our findings may only reflect situations where there is an obvious experience gap between team members.

Finally, our findings need to be understood within the context of a highly cooperative environment of Portal 2 where there is no competitive element. The presence of competition, either internal or external, may change the communication content and dynamics. To be able to design systems that encourage positive interactions, good teamwork and minimise toxicity, all potential input sources (i.e. individual characteristics, task characteristics and communication modalities) need to be investigated. Nonetheless, our findings on textual communication dynamics in Portal 2 shows that in this context, where teams are comprised of strangers in a one-off interaction, communication tends to be highly task-oriented and positive.

\section{Conclusion}

To better understand how ad hoc teams of strangers in digital games cooperate effectively, we investigated team communication. In contrast to the literature, we find that communication is negatively related to performance. Nonetheless, communication is an important factor of team performance above and beyond prior experience or expertise, accounting for $13 \%$ in variance explained. This suggests that teamwork between strangers can be improved by designing systems that facilitate more efficient communication. Team communication between strangers also tends to be more task-oriented. While in contrast to prior research on the communication content in digital game groups and teams, it more closely reflects communication in temporary teams in competitive cooperative games, zero-history computer-mediated work groups, and real-world 'swift starting action teams' like flight crews and crisis teams. Our study sheds light on the potential mechanisms through which communication influences performance and possibly, the development of social ties in teams of strangers. It moves us closer to the ultimate goal of designing systems that facilitate productive collaboration and positive experiences with strangers during play.

\subsection{Acknowledgement}

This work was supported by the EPSRC Centre for Doctoral Training in Intelligent Games Games Intelligence (IGGI) [EP/L015846/1]

\section{References}

[1] I. Simons and J. Newman, A History of Videogames. Carlton Books, Ltd., 2018.

[2] D. Williams, N. Ducheneaut, L. Xiong, Y. Zhang, N. Yee, and E. Nickell, "From tree house to barracks: The social life of guilds in world of warcraft," Games and culture, vol. 1, no. 4, pp. 338-361, 2006.

[3] I. Huvila, K. Holmberg, S. Ek, and G. Widén-Wulff, "Social capital in second life," Online Information Review, 2010.

[4] N. D. Poor, "Collaboration via cooperation and competition: Small community clustering in an mmo," in 2014 47th Hawaii International Conference on System Sciences, pp. 1695-1704, IEEE, 2014.

[5] G. Park, "With 10 million game pass subscribers, the xbox ecosystem begins to coalesce," 2020.

[6] newzoo, "Most popular core pc games," 2020.

[7] T. Wijman, "The global games market will generate $\$ 152.1$ billion in 2019 as the u.s. overtakes china as the biggest market," 2019.

[8] D. A. Harrison, S. Mohammed, J. E. McGrath, A. T. Florey, and S. W. Vanderstoep, "Time matters in team performance: Effects of member familiarity, entrainment, and task discontinuity on speed and quality," Personnel Psychology, vol. 56, no. 3, pp. 633-669, 2003.

[9] J. A. Espinosa, S. A. Slaughter, R. E. Kraut, and J. D. Herbsleb, "Familiarity, complexity, and team 
performance in geographically distributed software development," Organization science, vol. 18, no. 4, pp. 613-630, 2007.

[10] A. Zook, "Building matchmaking systems," Data Analytics Applications in Gaming and Entertainment, p. 33, 2019.

[11] Valve Corporation, "Portal 2," 2020.

[12] S. L. Marlow, C. N. Lacerenza, and E. Salas, "Communication in virtual teams: A conceptual framework and research agenda," Human Resource Management Review, vol. 27, no. 4, pp. 575-589, 2017.

[13] T. Driskell, J. E. Driskell, and E. Salas, "Lexicon as a predictor of team dynamics," in Team dynamics over time, Emerald Publishing Limited, 2017.

[14] M. A. Marks, J. E. Mathieu, and S. J. Zaccaro, "A temporally based framework and taxonomy of team processes," Academy of management review, vol. 26, no. 3, pp. 356-376, 2001.

[15] S. L. Marlow, C. N. Lacerenza, J. Paoletti, C. S. Burke, and E. Salas, "Does team communication represent a one-size-fits-all approach?: A meta-analysis of team communication and performance," Organizational Behavior and Human Decision Processes, vol. 144, pp. 145-170, 2018.

[16] R. F. Bales, "Interaction process analysis; a method for the study of small groups.," 1950.

[17] M. Hahsler and S. Koch, "Cooperation and disruptive behaviour-learning from a multi-player internet gaming community," in IADIS International Conference Web Based Communities, pp. 35-42, 2004.

[18] J. Peña and J. T. Hancock, "An analysis of socioemotional and task communication in online multiplayer video games," Communication research, vol. 33, no. 1, pp. 92-109, 2006.

[19] S. C. Herring, D. O. Kutz, J. C. Paolillo, and A. Zelenkauskaite, "Fast talking, fast shooting: Text chat in an online first-person game," in 2009 42nd Hawaii International Conference on System Sciences, pp. 1-10, IEEE, 2009.

[20] J. B. Walther, "Computer-mediated communication: Impersonal, interpersonal, and hyperpersonal interaction," Communication research, vol. 23, no. 1, pp. 3-43, 1996.

[21] E. H. Mckinney Jr, J. R. Barker, K. J. Davis, and D. Smith, "How swift starting action teams get off the ground: What united flight 232 and airline flight crews can tell us about team communication," Management Communication Quarterly, vol. 19, no. 2, pp. 198-237, 2005.

[22] F. R. Zijlstra, M. J. Waller, and S. I. Phillips, "Setting the tone: Early interaction patterns in swift-starting teams as a predictor of effectiveness," European Journal of Work and Organizational Psychology, vol. 21, no. 5, pp. 749-777, 2012.

[23] V. J. Shute, M. Ventura, and F. Ke, "The power of play: The effects of Portal 2 and Lumosity on cognitive and noncognitive skills," Computers \& Education, vol. 80, pp. 58-67, Jan. 2015.

[24] Z. O. Toups, J. Hammer, W. A. Hamilton, A. Jarrah, W. Graves, and O. Garretson, "A framework for cooperative communication game mechanics from grounded theory," in Proceedings of the first ACM SIGCHI annual symposium on Computer-human interaction in play, pp. 257-266, 2014.
[25] R. C. Wasserman and T. S. Inui, "Systematic analysis of clinician-patient interactions: a critique of recent approaches with suggestions for future research," Medical Care, pp. 279-293, 1983.

[26] J. L. Fleiss, "Measuring nominal scale agreement among many raters.," Psychological bulletin, vol. 76, no. 5, p. 378, 1971.

[27] J. R. Landis and G. G. Koch, "The measurement of observer agreement for categorical data," biometrics, pp. 159-174, 1977.

[28] S. W. Kozlowski, "Advancing research on team process dynamics: Theoretical, methodological, and measurement considerations," Organizational Psychology Review, vol. 5, no. 4, pp. 270-299, 2015.

[29] R. L. Helmreich, A. C. Merritt, and J. A. Wilhelm, "The evolution of crew resource management training in commercial aviation," The international journal of aviation psychology, vol. 9, no. 1, pp. 19-32, 1999.

[30] Z. O. Toups, A. Kerne, and W. A. Hamilton, "The team coordination game: Zero-fidelity simulation abstracted from fire emergency response practice," $A C M$ Transactions on Computer-Human Interaction (TOCHI), vol. 18, no. 4, pp. 1-37, 2011.

[31] D. Vaddi, Z. Toups, I. Dolgov, R. R. Wehbe, and L. Nacke, "Investigating the Impact of Cooperative Communication Mechanics on Player Performance in Portal 2," Canadian Human-Computer Communications Society, June 2016. Accepted: 2017-03-13T14:38:58Z.

[32] L. Eklund and M. Johansson, "Social play?: a study of social interaction in temporary group formation (pug) in world of warcraft," in DiGRA Nordic 2010: Experiencing Games: Games, Play, and Players (2010), First Nordic Digra August 16-17, 2010, Stockholm, Sweden, 2010.

[33] Y. Kou and X. Gui, "Playing with strangers: understanding temporary teams in league of legends," in Proceedings of the first ACM SIGCHI annual symposium on Computer-human interaction in play, pp. 161-169, 2014.

[34] S. J. Zaccaro, J. Gualtieri, and D. Minionis, "Task cohesion as a facilitator of team decision making under temporal urgency," Military Psychology, vol. 7, no. 2, pp. 77-93, 1995.

[35] K. Ames, B. Majolo, K. Hall, N. Wilson, R. Brumpton, and R. Garratt, "Human friendship favours cooperation in the iterated prisoner's dilemma," Behaviour, vol. 143, no. 11, pp. 1383-1395, 2006.

[36] C. J. Gersick and J. R. Hackman, "Habitual routines in task-performing groups," Organizational behavior and human decision processes, vol. 47, no. 1, pp. 65-97, 1990.

[37] J. R. Curhan and A. Pentland, "Thin slices of negotiation: Predicting outcomes from conversational dynamics within the first 5 minutes.," Journal of Applied Psychology, vol. 92, no. 3, p. 802, 2007.

[38] M. F. Jung, "Coupling interactions and performance: Predicting team performance from thin slices of conflict," ACM Transactions on Computer-Human Interaction (TOCHI), vol. 23, no. 3, pp. 1-32, 2016.

[39] F. Faul, E. Erdfelder, A.-G. Lang, and A. Buchner, " $G$ * power 3: A flexible statistical power analysis program for the social, behavioral, and biomedical sciences," Behavior research methods, vol. 39, no. 2, pp. 175-191, 2007. 